\title{
Heartbeat: Is it feasible to use computed tomographic coronary angiography for first-line diagnosis in chest pain patients?
}

doi:10.1136/heartjnl-2018-313443

Catherine M Otto

In a patient with recent onset chest pain symptoms, updated National Institute for Health and Care Excellence guidelines recommend computed tomographic coronary angiography (CTCA) as the initial diagnostic test when clinical features are consistent with typical or atypical angina or when atypical chest pain is accompanied by ECG changes suggesting ischaemia. Yet, it is not clear whether a CTCA study can be provided to all patients who meet these criteria. In this issue of Heart, Dreisbach and colleagues ${ }^{1}$ report that currently only about 42000 CTCAs are performed annually in the UK using 304 CTCA-capable scanners, interpreted by 198 accredited practitioners. They estimate that an additional 350000 scans (545 per 100000 population) would be required to fully implement these new guidelines (figure 1). Thus, there is a considerable shortage of both CTCA-capable scanners and qualified practitioners.

In an editorial, Cremer $^{2}$ expresses doubts about the recommendation to use CTCA as the first-line diagnostic test for suspected coronary artery disease, especially given that 'the UK is ill prepared both in terms of trained personnel and capable equipment. Given this predicament, despite increasing revascularisation, the failure of CTCA to demonstrate either superiority or non-inferiority for cardiac events should be emphasised.' 'With imaging, the value of a test is contingent upon technical considerations and diagnostic accuracy, and downstream treatment considerations, costs and outcomes. An appreciation for this hierarchy is especially important for common problems such as stable chest pain, given the far-reaching and costly implications' (figure 2).

Management of anti-thrombotic therapy is controversial in patients with atrial fibrillation (AF) after acute myocardial infarction (MI) or percutaneous coronary intervention (PCI). In a Danish nationwide registry of almost 3000 patients (mean age 76 years, $68 \%$ men) with $\mathrm{AF}$ after MI or PCI, all

Correspondence to Professor Catherine M Otto, Division of Cardiology, University of Washington, Seattle,WA 98195, USA; cmotto@uw.edu, cmotto@u. washington.edu

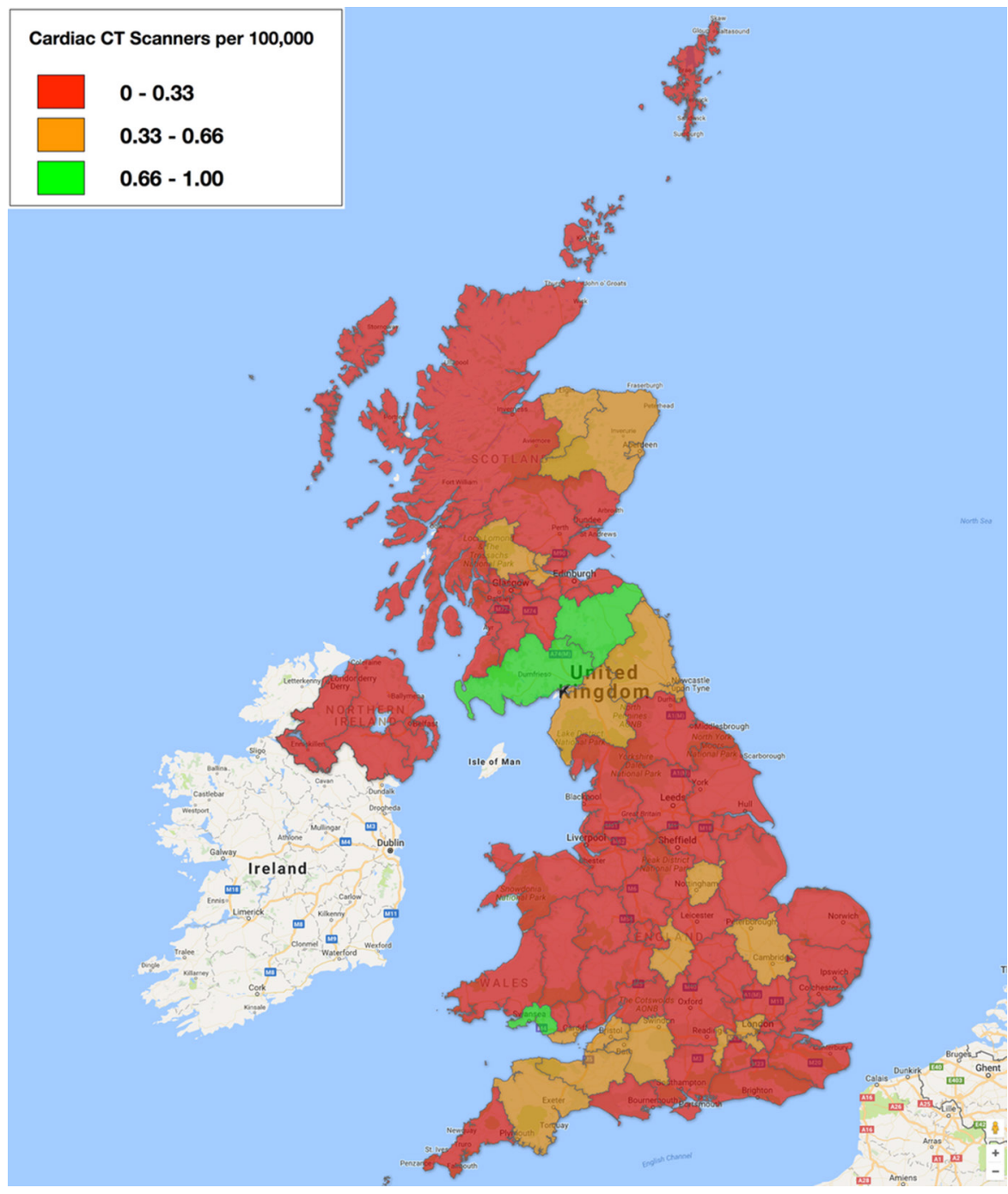

Figure 1 Map of the number of CT coronary angiography-capable scanners per 100000 population by health region in the UK.

received anti-platelet therapy with $2 / 3$ also being treated with vitamin- $\mathrm{K}$ antagonist anticoagulation and $1 / 3$ with a non-vitamin $\mathrm{K}$ antagonist oral anticoagulant (NOAC) medication. ${ }^{3}$ Over $95 \%$ of patients had a high $\mathrm{CHA}_{2} \mathrm{DS}_{2}$-VASc score and $32 \%$ had a high HAS-BLED score. From 2001 to 2016, the use of NOACs combined with anti-platelet therapy increased from $10 \%$ to $52 \%$ (figure 3 ).

Patti ${ }^{4}$ comments that "Triple antithrombotic therapy for a long time has been empirically considered the 'ideal' treatment in this setting of patients, as it may provide protection from both AF-related thromboembolic complications and ischaemic cardiac events, that is, oral anticoagulant therapy to prevent stroke or systemic embolism and dual antiplatelet therapy with aspirin plus clopidogrel to reduce MI and stent thrombosis. However, a close correlation between bleeding events and number or doses of antithrombotic agents has been clearly documented." "Moreover, 


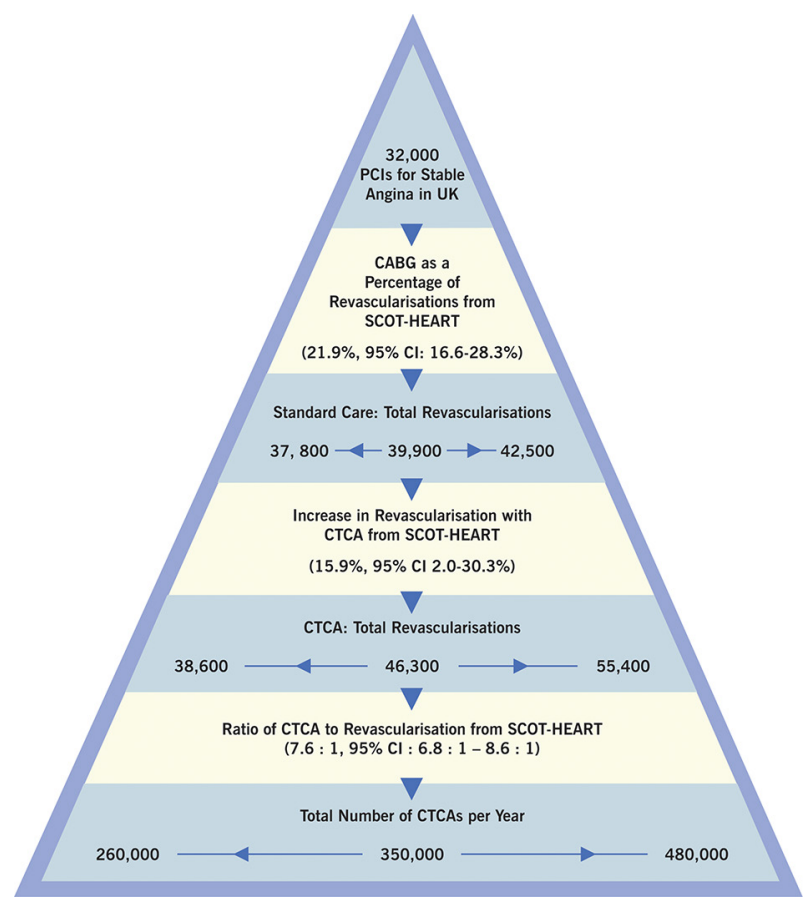

Figure 2 Crude calculations to express uncertainty in estimates for total CTCA increase in UK population based on SCOT-HEART sample. Bootstrap resampling $(n=1000)$ was performed to estimate $95 \% \mathrm{Cls}$ for the number of CABGs as a percentage of total revascularisations, the increase in revascularisation with CTCA from the intention-to-treat analysis and the ratio of CTCA to revascularisation from the as-treated analysis. The upper and lower bounds of these estimates were used in subsequent calculations to provide a general sense of the range of possible increases in CTCA volume as a result of implementation of the NICE guidelines. Analysis performed with R Core Team (2017). R: A language for environment and statistical computing. R Foundation for Statistical Computing, Vienna, Austria (URL https://www.R-project.org/). CABG, coronary artery bypass grafting; CTCA, CT coronary angiography; NICE, National Institute for Health and Care Excellence; $\mathrm{PCl}$, percutaneous coronary intervention.

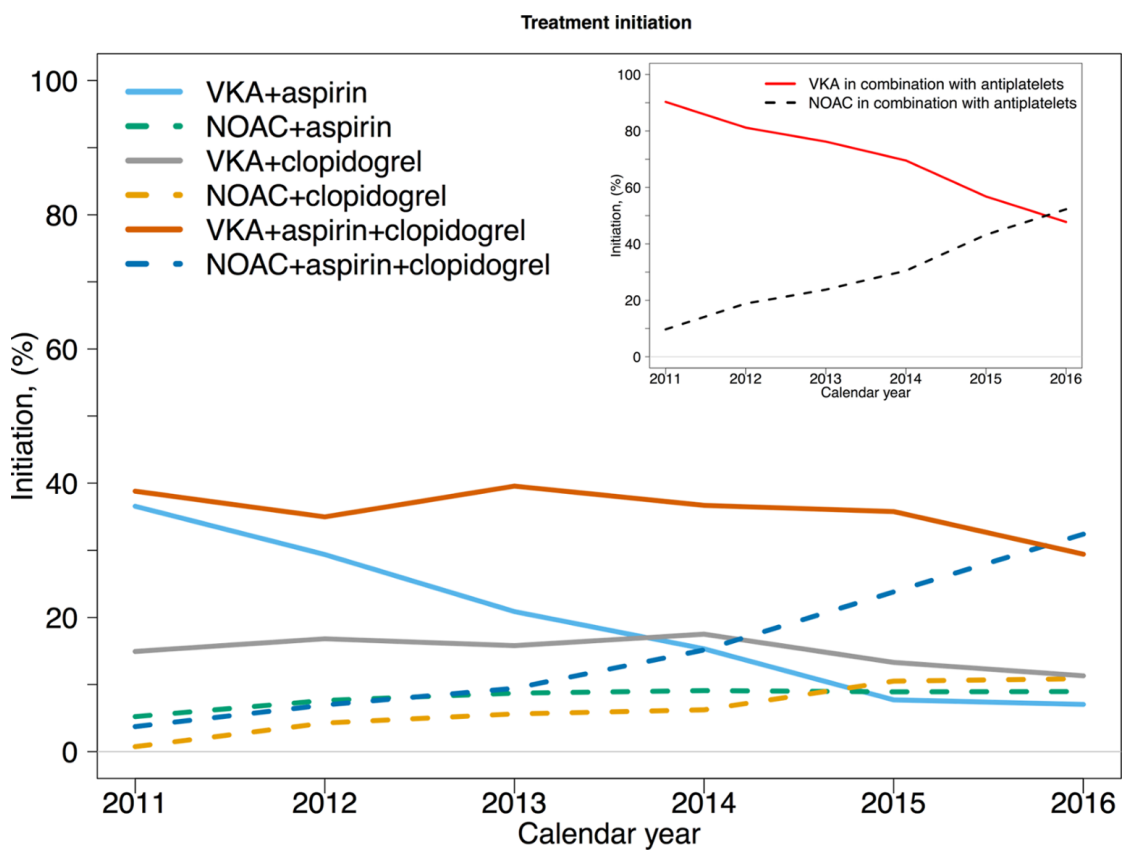

Figure 3 Treatment initiation according to treatment groups between August 2011 and September 2016. NOAC, non-vitamin K oral anticoagulant; VKA, vitamin K antagonist. no specific data on clinical outcome with the use of NOACs in this setting of patients were provided; this is a 'burning' unmet need, and future prospective, large-sized, real-world studies exploring such an issue are welcome."

The larger problem of AF prevention at the population level is addressed in an excellent review article by Boriani and Proietta ${ }^{5}$ in this issue of Heart. AF affects $0.5 \%$ of the world population, about 33.5 million people worldwide, and prevalence is increasing with the ageing of our population. Given the adverse clinical outcomes associated with $\mathrm{AF}$, measures to prevent this arrhythmia would be great value. It is hoped that $\mathrm{AF}$ incidence can be reduced by attention to risk factors, including reducing weight and increasing physical activity, as well as ensuring optimal therapy for hypertension and diabetes (figure 4). 'The AF epidemic is becoming a worldwide threat and, in this complex and evolving scenario, it is worth considering a paradigm shift for healthcare interventions aimed at reducing the clinical and economic consequences of AF. This could imply overcoming the conventional 'reactive' approaches to healthcare and invest resources for more 'proactive' initiatives, in the context of 'primordial' prevention, to be applied at a community level, as well as in the context of primary prevention to be applied at an individual level.'

The Education in Heart ${ }^{6}$ article in this issue reviews the indications and technique for myocardial biopsy. The primary indication for myocardial biopsy is monitoring for rejection after heart transplantation. Other indications include new onset fulminant heart failure, recent onset ventricular arrhythmias, atrioventricular block or refractory heart failure. In these situations, myocardial biopsy may provide evidence of giant cell, eosinophilic or lymphocytic myocarditis; granulomatous myocarditis; or sarcoidosis. However, biopsy data are only one factor in clinical decision making in these patient groups, along with advanced imaging, clinical and laboratory data.

Competing interests None declared.

Patient consent Not required.

Provenance and peer review Commissioned; internally peer reviewed.

(C) Article author(s) (or their employer(s) unless otherwise stated in the text of the article) 2018. All rights reserved. No commercial use is permitted unless otherwise expressly granted.

\section{A) Check for updates}

To cite Otto CM. Heart 2018;104:872-874.

Heart 2018:104:872-874

doi:10.1136/heartjnl-2018-313443 


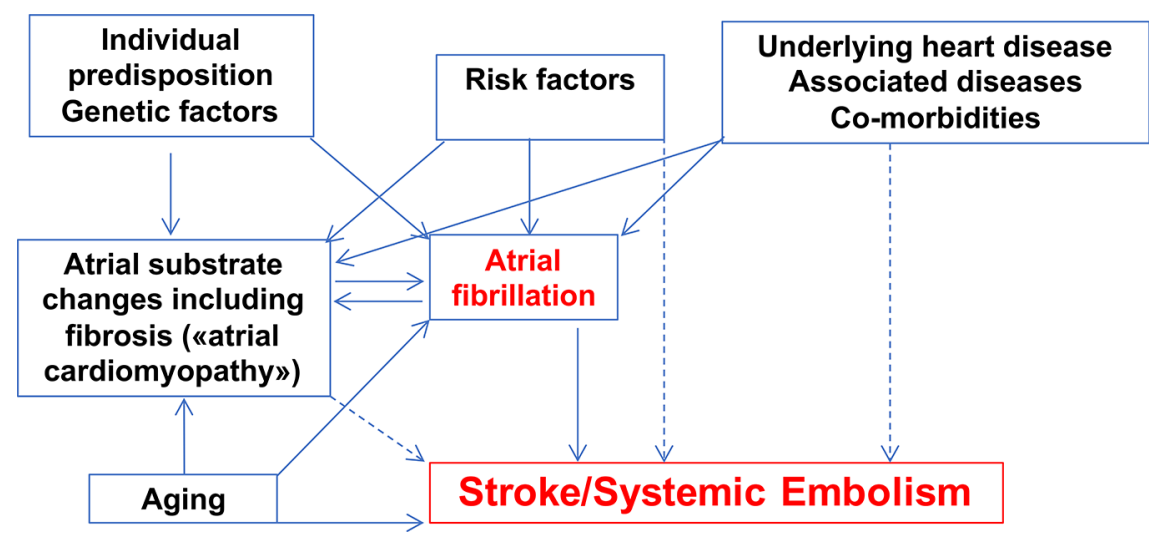

Figure 4 The complex interplay between risk factors, atrial and cardiac substrates, ageing and predisposing factors that is object of interventions for prevention of atrial fibrillation and of stroke/ systemic embolism.

\section{REFERENCES}

1 Dreisbach JG, Nicol ED, Roobottom CA, et al. Challenges in delivering computed tomography coronary angiography as the first-line test for stable chest pain. Heart 2018;104:921-7.

2 Cremer PC. Compounding uncertainties with first-line computed tomography coronary angiography. Heart 2018:doi: 10.1136/heartjnl-2017-312572 [Epub ahead of print].

3 Sindet-Pedersen C, Staerk L, Lamberts M, et al. Use of oral anticoagulants in combination with antiplatelet(s) in atrial fibrillation. Heart 2018:104:912-20.

4 Patti G. Patients with atrial fibrillation and coronary events: are we any closer to coming out from the labyrinth of the various antithrombotic strategies? Heart 2018;104:878-9.

5 Boriani G, Proietti M. Atrial fibrillation prevention: an appraisal of current evidence. Heart 2018;104:882-7.

6 Francis R, Lewis C. Myocardial biopsy: techniques and indications. Heart 2018;104:950-8. 